\title{
THE MECHANISM OF ACTION OF THE TUMOUR SUPPRESSOR GENE PTEN
}

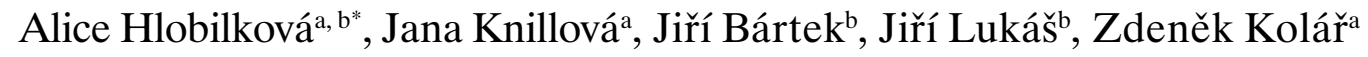 \\ a Institute of Pathology and Laboratory of Molecular Pathology, Faculty of Medicine, Palacký University, Hněvotínská 3, \\ 77515 Olomouc, Czech Republic, e-mail: hlob@hotmail.com \\ ${ }^{b}$ Cell Cycle and Cancer, Danish Cancer Society, Copenhagen, DK-2100, Denmark
}

Received: May 17, 2003; Accepted in revised form June 26, 2003

Key words: PTEN/ Tumour suppressor / PI3-K / PKB/Akt / ERK-MAPK / FAK / Cell cycle regulation

Intracellular levels of phosphorylation are regulated by the coordinated action of protein kinases and phosphatases. Disregulation of this balance can lead to cellular transformation. Here we review knowledge of the mechanisms of one protein phosphatase, the tumour suppressor PTEN/MMAC/TEP-1 apropos its role in tumorigenesis and signal transduction. PTEN plays an important role in the phosphatidyl-inositol-3-kinase (PI3-K) pathway by catalyzing degradation of phosphatidylinositol-(3,4,5)-triphosphate generated by PI3-K. This inhibits downstream targets mainly protein kinase B (PKB/Akt), cell survival and proliferation. PTEN contributes to cell cycle regulation by blockade of cells entering the S-phase of the cell cycle, and by upregulation of $\mathrm{p} 27^{\mathrm{Kip} 1}$ which is recruited into the cyclin E/cdk2 complex. PTEN also modulates cell migration and motility by regulation of the extracellular signalrelated kinase - mitogen activated protein kinase (ERK-MAPK) pathway and by dephosphorylation of focal adhesion kinase (FAK). We also emphasize the increasingly important role that PTEN has from an evolutionary point of view. A number of PTEN functions have been elucidated but more information is needed for utilization in clinical application and potential cancer therapy.

\section{INTRODUCTION}

PTEN (phosphatase and tensine homolog deleted on chromosome ten)/MMAC (mutated in multiple advanced cancers) has been identified simultaneously by two research groups as a candidate tumour suppressor gene located at $10 \mathrm{q} 23^{1,2}$. Another group identified the same gene in the search for new dual-specificity phosphatases and named it TEP-1 (TGF- $\beta$ regulated and epithelial cell-enriched phosphatase) ${ }^{3}$. Overall, PTEN is one of the most common targets of mutation in human cancer, with a mutation frequency approaching that of p53. PTEN is also mutated in inherited cancer predisposition disorders.

A variety of cellular functions have been reported for PTEN using different experimental model organisms; regulation of cell division, cell survival, apoptosis and cell migration in human and mouse cells, involvement in the dauer formation in Caenorhabditis elegans, regulation of cell size in Drosophila eye development and having a role in sporulation in Saccharomyces cerevisiae ${ }^{4}$.

The PTEN cDNA encodes a 403-amino-acid peptide with relative molecular mass of $55 \mathrm{kD}^{1,3,5,6,7}$. Two major transcripts of approximately 2 and $5 \mathrm{~kb}$ and several other minor RNA species have been detected in a wide variety of cell lines and immunological in situ detections indicate different localisation of the PTEN protein $^{8}$. In xenografts from prostate tumour tissue were observed exclusively cytoplasmic staining, which supports the suggested role of PTEN as a signal transduction molecule connecting the cytoskeleton to intracellular signaling pathway ${ }^{9}$. Immunohistochemical analysis of tissue sections from a group of glioblastomas (GBMs) shows perinuclear cytoplasmic and nuclear staining ${ }^{10}$. Two distinct patterns of positive staining were observed. All of the tumour cells were either homogenously or heterogenously stained for PTEN. Positivity of PTEN was also present in vascular endothelial cells and neurons. In archival paraffin tissue section from resected prostate cancer PTEN expression was seen in the cytoplasm of the secretory cells with a granular staining pattern $^{11}$.

\section{STRUCTURE OF THE PTEN MOLECULE IN RELATION TO ITS FUNCTION}

The crystal structure study of PTEN reveals two major domains: an $\mathrm{N}$-terminal phosphatase domain consisting of five stranded $\beta$-sheets surounded by six $\alpha$-helices, connected via a flexible internal loop to the C-terminal domain consisting of two antiparallel $\beta$-sheets linked by two short $\alpha$-helices ${ }^{12}$. The $\mathrm{N}$-terminal domain with 179-residues (residues 7-185) has significant homology to the cytoskeletal protein tensine and auxilin. Tensine binds to actin and participates in the assembly of the signaling complex at focal adhesion, downstream 
of which lie pathways that regulate cellular integrity, cell-to-cell communication, cell-microenvironment interactions and cell migration. Auxilin is involved in the transport of synaptic vesicles ${ }^{8}$.

The 166 residues of $\mathrm{C}$-terminal domain contains the protein tyrosine phosphatase (PTP) motif and has a structure similar the dual specifity phosphatase (DSP). The phosphatase active site of PTEN with its basic charge is important for Phosphatidyl-Inositol-3,4,5-tri-Phosphate (Ptd-Ins-3,4,5-P ) binding (Fig. 1). The C-terminal has a structure similar to the $\mathrm{C} 2$ domain that mediates the $\mathrm{Ca}^{+2}$-dependent membrane recruitment of several signaling proteins. The PTEN C2 domain lacks the $\mathrm{Ca}^{+2}$ ligands, and in this respect it is similar to the $\mathrm{C} 2$ domains of the $\mathrm{Ca}^{+2}$ - independent protein kinase $\mathrm{C}$ (PKC) isotypes. The in vitro membrane binding activity of PTEN does not require $\mathrm{Ca}^{+2}$. It is strongly suggested that PTEN binds to phospolipid membranes and its lipid binding activity is important for its tumour suppressor function. The $\mathrm{C} 2$ and phosphatase domains associate across an extensive interface that is adjacent to the phosphatase active site and consists of conserved residues frequently mutated in cancer (Fig. 1, 2). The PTEN C2 domain may help recruit PTEN to the membrane and also serve to position and orient the catalytic domain optimally with respect to the membrane-bound substrate ${ }^{13}$.

The PTEN HCXXGRXXR motif forms a loop (P-loop, residues 123-130) located at the bottom of the active site pocket, in common with PTPs and DSPs (Fig. 1). In the PTEN HCXXGRXXR motif the Cys-124

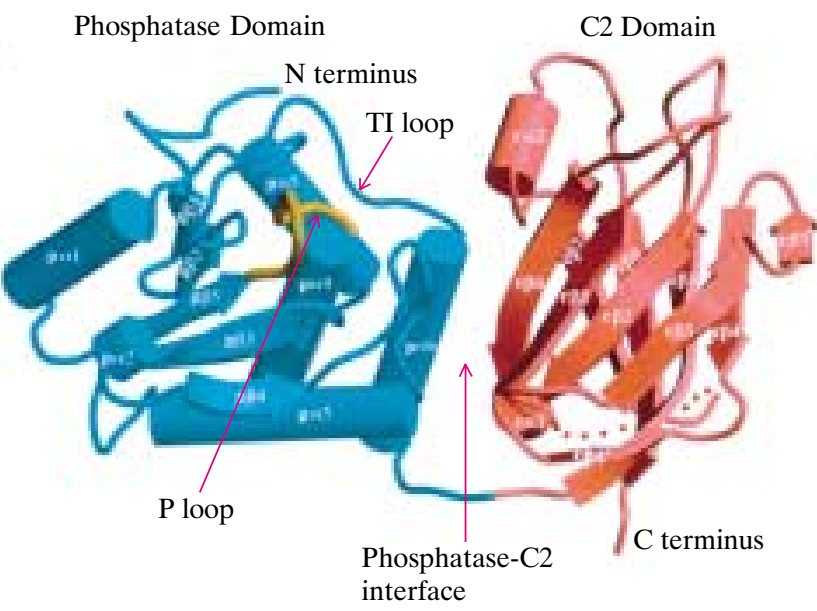

Fig. 1. The crystal structure of PTEN with phosphatase domain, $\mathrm{C} 2$ domain, phosphatase-C2 interface, TI loop and P loop. Adapted according to the article: Crystal structure of the PTEN tumour suppressor: implications for its phosphoinositide phosphatase activity and membrane association (Cell 99, 323-334, 1999). and Arg-130 residues are essential for catalysis and the His-123 and Gly-127 residues are important for the conformation of the $\mathrm{P}$ loop $^{14}$. The PTEN has a second anion-binding site adjacent to the catalytic phosphatebinding site, called the TI loop (Fig. 1). Mutations of Thr-167 or Gln-171 on the TI loop, Lys-125 in the conserved basic residues in the PTEN active site motif and His-93 resulted in reductions of enzymatic activity toward Ptd-Ins-3,4,5- $\mathrm{P}_{3}$ about $75 \%{ }^{13}$.

The C-terminus of PTEN has a consensus binding motif - postsynaptic density protein (PSD95)/Drosophila disc large tumour suppressor (dlg)/tight junction protein ZO1 (PDZ-binding motif) and this may interact with PDZ domain-containing protein $\mathrm{s}^{15}$. The PDZ binding site of PTEN is represented by the aminoacids: Ile-Thr-Lys-Val and it is unnecessary for the function of some systems ${ }^{16,17}$. Other studies have shown that PDZ binding site associates with members of membrane-associated guanylate kinases (MAGI). In the absence of an interaction between PTEN and MAGI 2 or MAGI 3 , the ability of PTEN to decrease Akt phosphorylation is impaired, suggesting that the PDZ binding site contributes to the PTEN function in some models ${ }^{17,18,19,20}$. In human breast cancer tissue a stop codon mutation in PTEN has been identified that adds a short carboxyl extension, destroying the PDZ binding site in PTEN ${ }^{21}$. It has recently been shown that an interaction of PDZ domain with MAGI-2 stabilises the PTEN protein and enhances its activity, and threonine phosphorylation of the PDZ-binding motif both inhibits and stimulates PDZ-binding ${ }^{12}$.

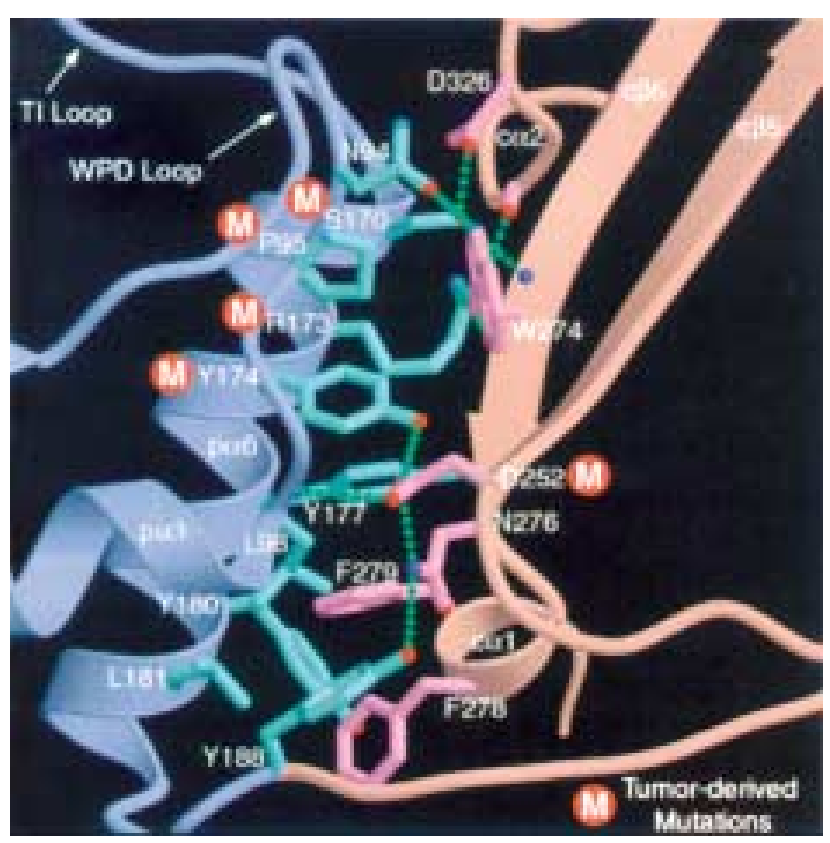

Fig. 2. Phosphatase-C2 interface with many residues participating in hydrogen binding networks have been found mutated in tumours. Adapted according to the article: Crystal structure of the PTEN tumour suppressor: implications for its phosphoinositide phosphatase activity and membrane association (Cell 99, 323-334, 1999). 
Several growth factors induce PTEN phosphorylation, probably at tyrosine residues 240,315 , and 336 . This is followed by decreased protein stability and enzymatic activity $^{21}$. A large number of PTEN nonsense or frameshift mutations found in tumours are targeted to the C-terminal domain of the protein, suggesting an important role for this domain in the regulation of the PTEN tumour suppressor activity. C-terminal PTEN domain is rich in phosphorylation sites, and phosphorylation of the PTEN C terminus has been recently reported to affect PTEN protein stability and function. It is constitutively phosphorylated by protein casein kinase CK2 at Ser 370 and Ser 385 residues. This suggests the role of phosphorylation as a regulatory mechanism of PTEN biological activity by modulating its stability to proteasome-mediated degradation. However it is still not proven that the stability of the protein can reflect the localization of PTEN within a subcellular compartment ${ }^{22}$.

Two novel alternatively spliced transcripts of the human PTEN gene, encoding protein products with altered C-terminal amino acid sequences in comparison with full length PTEN have been identified. PTEN- $\Delta$ is generated by inclusion of the 5 end of intron $\mathrm{H}$ between exon 8 and 9, resulting in a truncated coding sequence. PTEN-B is generated by alternative splicing of the end of exon 5. The generated open reading frame is truncated, and it encounters a stop codon after only five aminoacids. The coexpression of full length PTEN and two splicing variants have been demonstrated in normal lymphocytes and in non-malignant prostate cell lines. PTEN- $\Delta$ and PTEN-B are likely to be short-lived structures with limited phosphatase activity. The low-stability of PTEN protein homologues could imply they act as competitors for substrates or they could be stabilised by interaction with other molecular structures ${ }^{12}$.

\section{PHOSPHATASE ACTIVITY}

The level of phosphorylation within cells is tightly regulated by the action of protein kinases and protein phosphatases. Disregulation of the activity of either of these players can lead to cellular tranformation. Many protein tyrosine kinases are proto-oncogenes and it has been postulated that some protein phosphatases may act as tumour suppressors. PTEN is a member of the PTP gene superfamily. PTP superfamily members can be further subdivided into classic PTP and dual specificity phosphatase families, comprising: $\operatorname{cdc} 25 \mathrm{~A}, \mathrm{~B}$, and $\mathrm{C}$ (enzymes that specifically dephosphorylate and activate cyclin-dependent kinases), BVP (a baculovirus phosphatase), human VHR (a protein related to the vaccinia virus VH1 phosphatase), MKP1 (MAP kinase phosphatase 1 , an enzyme that specifically dephosphorylates and activates mitogen activated protein kinase MAPK), the yeast cdc14 (an enzyme involved in the control of cell cycle progression), and the family of prenylated protein tyrosine phosphatases PRL1, 2, and 3. PTEN is a dual specificity protein phosphatase, dephosphorylates phosphotyrosine, phosphoserine, and/or phosphothreonine. It has phosphoinositide 3-phosphatase activity and plays an important role in the modulation of the phosphatidylinositol 3-kinase (PI3-K) pathway ${ }^{8}$. Overexpression of PTEN reduces insulin induced Ptd-Ins-3,4,5- $\mathrm{P}_{3}$ without affecting insulin-induced PI3-K activation. Transfection of the catalytically inactive mutant of PTEN (C124S) causes Ptd-Ins-3,4,5- $\mathrm{P}_{3}$ accumulation in the absence of insulin stimulation. PTEN catalyzes dephosphorylation of Ptd-Ins-3,4,5- $\mathrm{P}_{3}$ at position 3 on the inositol ring. Ptd-Ins-3,4,5- $\mathrm{P}_{3}$ is an important second messenger involved in cell growth signaling. It is specifically produced from Ptd-Ins-(4,5)- $\mathrm{P}_{2}$ by $\mathrm{PI} 3-\mathrm{K}$ upon stimulation by a variety of ligands (Fig. 3). Ptd-Ins$3,4,5-\mathrm{P}_{3}$ can directly activate protein kinase $\mathrm{B}(\mathrm{PKB} /$ Akt), which regulates cell survival and cell proliferation (Fig. 3). PTEN has Ptd-Ins-3,4,5-P 3 -phosphatase activity and may act in vivo as a regulator of Ptd-Ins$3,4,5-\mathrm{P}_{3}$, which produces a substrate that can be recycled by PI3-K ${ }^{15}$ (Fig. 3).

\section{PI 3-K, PKB/Akt SURVIVAL SIGNALING PATHWAY}

Several papers establish a link between the PI3-K/ Akt pathway and human cancers via defects in PTEN 23 . Association with PI3-K and elevated levels of specific lipid products of the enzyme Ptd-Ins-3,4,5- $\mathrm{P}_{3}$ correlate with the transforming ability of several oncoproteins. Growth factor stimulation and oncoprotein transformation correlate with increase in vivo levels of Ptd-Ins3,4- $\mathrm{P}_{2}$ and Ptd-Ins-3,4,5- $\mathrm{P}_{3}$. These lipids are absent from quiescent cells but appear within seconds to minutes of stimulation with growth factors, platelet derived growth factor (PDGF), nerve growth factor (NGF), or insulinlike growth factor (IGF-1). These lipids act as membrane-embedded second messengers. A variety of cytosolic signaling proteins have evolved the ability to bind to one or both of these lipids as a mechanism of recruitment to the membrane ${ }^{24}$. Akt was discovered as the product of a retrovirus-encoded oncogene that transforms lymphoid cells $^{25}$. There are three mammalian isoforms of Akt: Akt $1, A k t 2$ and $A k t 3{ }^{26}$. The protein products have a catalytic domain with close similarity to the protein kinase A (PKA) and PKC family of protein kinases, and Akt has been independently termed PKB as a PKC homologue $^{27}$. The first evidence that $\mathrm{PKB} / \mathrm{Akt}$ is regulated via a pathway involving PI3-K came from studies of PDGF-dependent PKB/Akt activation in fibroblasts ${ }^{28}$. The N-terminal PH domain of PKB/Akt acts as an autoinhibitory domain. This domain has a high affinity for Ptd-Ins-3,4- $\mathrm{P}_{2}$ and Ptd-Ins-3,4,5- $\mathrm{P}_{3}$ Binding to these phosphoinositides localizes PKB/Akt to the membrane and opens up the catalytic site. Protein-serine/threonine kinase, PDK1, also has the PH domain that binds PtdIns-3,4- $\mathrm{P}_{2}$ and Ptd-Ins-3,4,5- $\mathrm{P}_{3}$ tightly, allowing it to colocalize with $\mathrm{PKB} / \mathrm{Akt}$ and phosphorylate the activation loop. The full function of PKB/Akt requires phosphorylation at a second $\mathrm{C}$-terminal site by a distinct kinase. 
Activation of PI3-K and PKB/Akt has been shown to provide a survival signal in response to NGF, IGF-1, PDGF, interleukin-3 (IL-3) and the extracellular matrix $^{29}$. $\mathrm{PKB} / \mathrm{Akt}$ is likely to send survival signals by phosphorylating multiple targets, including the Bcl-2 family member Bad rendering it incapable of blocking Bcl-2 or Bcl- $\mathrm{x}_{\mathrm{L}}$ activity $^{30}$ and the cell death pathway enzyme caspase-931 (Fig. 3). PKB/Akt catalyzes phosphorylation of another serine/threonine kinase, glycogen synthase kinase 3 (GSK3), and this results in GSK3 inhibition ${ }^{32}$ (Fig. 3). GSK3 promotes cyclin D proteolysis. Thus by catalyzing GSK3 inhibition PKB/Akt may contribute to cyclin D accumulation and cell cycle entry ${ }^{33}$. The forkhead transcription factors FKHR, FKHRL1 and the protein-serine/threonine kinase $\mathrm{p} 70^{\mathrm{S} 6 \text { kinase }}$ are also activated by the PI3-K pathway (Fig. 3). The forkhead transcription factors are inactive in PTEN null cells. Reconstitution of FKHR activity, in the absence of PTEN, can induce both cell cycle arrest and apoptosis in PTEN null cells ${ }^{34,35}$. P70 ${ }^{\text {S6kinase }}$ may contribute to cell growth by regulating translation of key mRNAs ${ }^{36}$. Normally, $\mathrm{PKB} / \mathrm{Akt}$ activity is low in the absence of growth factor stimulation. PTEN-deficient tumor cell lines, as well as immortalized fibroblasts and tumours derived from PTEN-deficient mice, exhibit high basal levels of PKB/Akt phosphorylation. Reconstitution of wild-type (wt) PTEN expression restores normal PKB/Akt regulation ${ }^{23}$. PTEN action can be overcome by expression of the constitutively active form of $\mathrm{PKB} / \mathrm{Akt}$. These results indicate that deregulation of the PI3-K/PKB/Akt signal transduction pathway may contribute to a large percentage of human cancers.

\section{ROLE IN CELL CYCLE REGULATION}

Overexpression of wt PTEN in MCF-7 breast cancer cell line leads to the suppression of cell growth through the blockade of cell cycle progression ${ }^{37}$, an increased abundance of cyclin dependent kinase (cdk) inhibitor $\mathrm{p} 27^{\mathrm{Kip} 1}$, a decrease in the protein level of cyclin $\mathrm{D}_{1}$ and inhibition of $\mathrm{PKB} / \mathrm{Akt}$ phosphorylation. Expression of the phosphatase-dead PTEN mutant, C124S, has the opposite effect on the abundance of $\mathrm{p} 27^{\mathrm{Kip} 1}$, cyclin $\mathrm{D}_{1}$ and PKB/Akt phosphorylation. The G129E mutant which does not have lipid phosphatase activity but retains protein phosphatase activity behaves like C124S except for a decreasing cyclin $\mathrm{D}_{1}$ level similar to that of wt PTEN. The lipid phosphatase branch of PTEN upregulates $\mathrm{p} 27^{\mathrm{Kip} 1}$ by blocking $\mathrm{PI} 3-\mathrm{K}$ signaling, whereas the protein phosphatase branch down-regulates cyclin $\mathrm{D}_{1}$. Furthermore, either estrogen or insulin abrogates PTEN-mediated upregulation of $\mathrm{p} 27^{\mathrm{Kip} 1}$ and partially blocks PTEN-mediated growth supression. In contrast, the combination of estrogen and insulin eliminates the upregulation of $\mathrm{p} 27^{\mathrm{Kip} 1}$, downregulation of cyclin $\mathrm{D}_{1}$ and it completely blocks PTEN-mediated growth suppres$\operatorname{sion}^{38}$. In glioblastoma cell lines, $\mathrm{p} 27^{\mathrm{Kip} 1}$ is recruited into cyclin E/cdk2 immunocomplexes when PTEN is over- expressed in PTEN-mutant, but not in PTEN wt cells. The recruitment of $\mathrm{p} 27^{\mathrm{Kip} 1}$ to this complex leads to reduction in cyclin E/cdk2 kinase activity by $80 \%$, an overall diminution of phosphorylation levels in endogenous retinoblastoma protein $(\mathrm{pRb})$ and $\mathrm{G}_{1}$ cell cycle arrest. These effects culminate in a substantial reduction in the number of cells reaching the S-phase of the cell cycle. Cdk4-associated kinase activity, levels of $\mathrm{p} 21^{\mathrm{Waf} 1 / \mathrm{Cip} 1}$ and cyclin $\mathrm{E}$ in immuno-complexes were unaffected $^{39}$.

\section{ERK-MAPK SIGNALING PATHWAY AND ROLE IN REGULATION OF CELL MOTILITY}

It was discovered that PTEN can exert a growth suppressive effect by blocking insulin-stimulated phosphorylation of mitogen-activated protein kinase (MAPK). The Ras/MAPK pathway is the central signal transduction pathway involved in the regulation of a broad range of cellular functions. Well-defined nuclear targets of this pathway include a member of the Ets family of transcription factors, ETS-2. Its phosphorylation on the threonine residue 72 in Ras-dependent fashion leads to an increase in ability to transactivate target genes. ETS-2 expression correlates with cell proliferation and it can activate the promoter of cyclin $\mathrm{D}_{1}$, a key positive regulator of the $G_{1} / S$ cell cycle progression. Induction of wt PTEN in the MCF-7 cell line

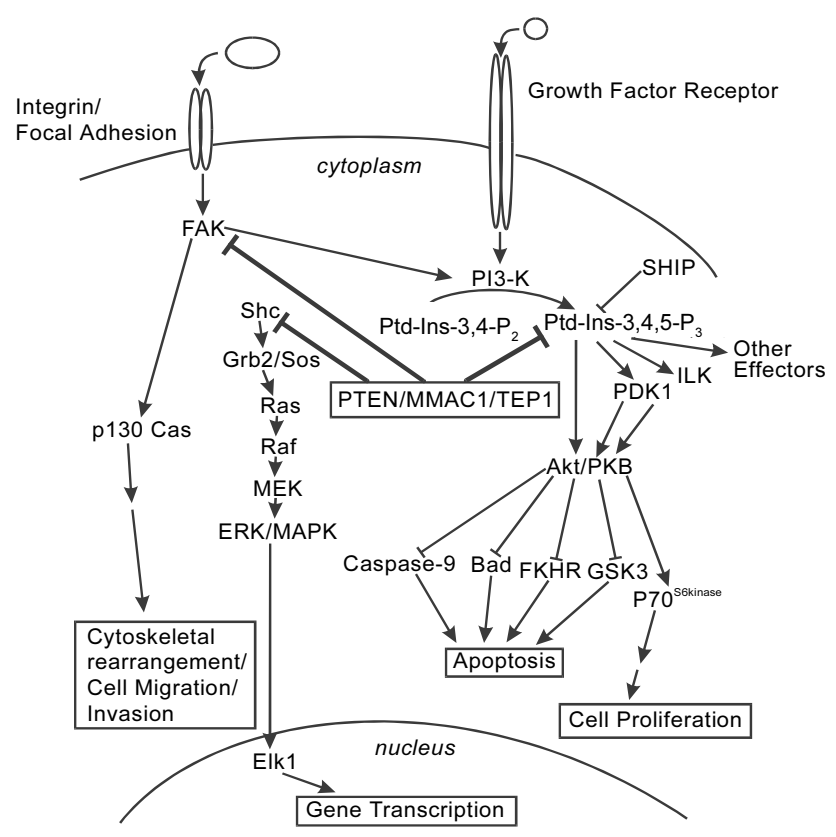

Fig. 3. Proposed model for PTEN/MMAC1/TEP1 mode of action. Schematic view of the known targets of negative regulation by PTEN, either via its protein tyrosine phosphatase activity or its phosphoinositide 3-phosphatase activity. Adapted according to the article: PTEN in signal transduction and tumorigenesis (Eur. J. Biochem. 263, 605-611, 1999). 
resulted in marked decrease in phosphorylation levels of ETS-2, PKB/Akt, and extracellular signal-related kinase (ERK) members of the MAPK family ERK1/2, in contrast to the phosphatase-dead C124S PTEN mu$\operatorname{tant}^{40}$. PTEN may block the MAPK pathway at some point upstream of MAPK or ERK (MEK) that might be common to both the integrin- and growth factor-mediated signaling pathway. PTEN most likely does not directly dephoshorylate ETS-2, but instead acts upstream of ETS-2 and ERK, and thus indirectly inhibits ETS-2 phosphorylation. Ras has been suggested as an initial component between integrin- and growth factor-mediated MAPK signaling pathways. The ability of PTEN to inhibit both integrin- and growth factor-mediated MAPK signaling pathways might be due to PTEN inhibiting Ras activation (Fig. 3). Upstream MEK, Ras activation, $\mathrm{SH}_{2}$-phosphotyrosine-binding adapter protein (Shc) phosphorylation, and its interaction with the adapter protein $\mathrm{Grb}_{2}$ are inhibited, but the EGF receptor, c-Jun $\mathrm{NH}_{2}$-terminal kinase (JNK) and PKB/Akt phosphorylation are not affected (Fig. 3). The Ras inhibition can be overcome by expression of activated Ras and overexpression of an activated downstream component of the pathway, MEK1, can antagonize the biological effect of PTEN on cell spreading and focal contact formation $^{41}$. It has been shown that the G129E mutant of PTEN, which lacks lipid phosphatase activity but retains protein phosphatase activity, can still inhibit integrin-mediated cell migration, spreading, focal adhesions, and tumour cell invasion, whereas protein and lipid phosphatase dead mutant $\mathrm{C} 124 \mathrm{~S}$ of PTEN can not, demonstrating an important role of protein phosphatase activity in PTEN function. PTEN directly associates with the focal adhesion kinase (FAK) and can reduce its tyrosine phosphorylation as well as its potential downstream effector, p130 Crk-associated substrate (p130 ${ }^{\mathrm{Cas}}$ ) (Fig. 3). Furthermore, overexpression of FAK or p130 Cas can antagonize the effects of PTEN on cell migration and invasion. MAPK activation may partially antagonizes PTEN function with partial rescue of cell spreading on fibronectin impaired by PTEN. This inhibition of PTEN was associated with effects on Shc phosphorylation. Shc links tyrosine kinases to Ras signaling by recruiting the $\mathrm{Grb}_{2}$-Sos complex to the plasma membrane in a tyrosine phosphorylation-dependent manner. PTEN can inhibit tyrosine phosphorylation of both FAK and Shc (Fig. 3). Both proteins are implicated in integrin signaling and both can bind and potentially activate the Ras-MAPK pathway. PTEN, Shc and FAK regulate cell movement through two different mechanisms: one is the pathway from Shc through the MAPK pathway leading to the stimulation of random cell motility, and the other is from FAK through $130^{\text {Cas }}$ leading to stimulation of directionally persistent cell migration. These two pathways are counterbalanced and integrated with the actions of PTEN ${ }^{42}$.

\section{STRUCTURE AND FUNCTION AT DIFFERENT EVOLUTIONARY LEVELS}

One homolog of mammalian PTEN found in Caenorhabditis elegans is abbreviated as CePTEN. The amino-terminal region of CePTEN contains the phosphatase catalytic domain, and this region shares $38 \%$ sequence identity with the corresponding domain of human PTEN. The carboxyl-terminal region of CePTEN has lower levels of sequence homology than to human PTEN. CePTEN is involved in dauer formation and lifespan regulation and is encoded by the daf- 18 gene. The dauer state is essentially a state of hibernation that is normally only entered under conditions of starvation or overcrowding. The nr2037 CePTEN mutation reveals a deletion that removes 990 nucleotides of the gene spanning parts of intron 2 and exon 3 . This mutation abolishes the phosphatase activity of CePTEN. A strain homozygous form nr2037 is viable and shows no obvious abnormalities in development or morphology when grown under well fed conditions. The daf-18 nr2037 homozygotes at high-saturation growth densities or under starvation conditions failed to form dauers (a dauer defective phenotype). The dauer defective phenotype can be rescued by germ-line transformation with the wt CePTEN gene. Daf-18 nr2037 mutants have a shorter lifespan than wt animals by about $30 \%$. This provides genetic evidence that CePTEN/daf-18 is one of the rate-limiting factors controlling the onset of aging in Caenorhabditis elegans ${ }^{43}$.

The PTEN gene homologue, TEP1, exists in the budding yeast Saccharomyces cerevisiae. In the absence of a functional TEP1 gene, yeast cells grow normally, but homozygous diploids display resistence to the PI3-K inhibitor wortmanin and lithium ions. Neither TEP1 haploids nor diploids have altered life spans. TEP1 RNA is present throughout the cell cycle, and levels are dramatically upregulated during meiotic development. The spores produced in homozygous TEP1 mutants indicate a specific defect in the trafficking or deposition of dityrosine, a major component of yeast spore walls, on the surface. The maturation of the spore wall and its connection to the meiotic cell is analogous to the interaction of epithelial cells with the extracellular matrix. Perturbation of these cell contacts is important in carcinogenesis and suggests that analysis of TEP1 in yeast will be directly applicable to understanding the role of its homolog PTEN as a tumour suppressor in humans ${ }^{44}$.

The Drosophila (dPTEN) and human PTEN gene product are similar in length and share $44 \%$ sequence similarities in both the N-terminal phosphatase domain and the C-terminal region. Both dPTEN defective heterozygotes and homozygotes die in the early larval stage. The size of the dPTEN mutant cells in the eye are much larger then neighboring wt cells. Overexpression of PTEN in proliferating cells of the eye disc results in dramatic reduction of eye size in a dose-dependent manner. Even multiple copies of PTEN can completely 
eliminate the eye. Flow cytometry analysis after PTEN overexpression shows the cell cycle arrest in the $G_{2}$ or $\mathrm{G}_{2} / \mathrm{M}$ phase in proliferating cells and cell death analysis shows apoptosis in differentiating cells during eye development. The small eye phenotype of PTEN overexpression is suppressed by overexpression of wt Drosophila PI3-K (D-PI3-K) and enhanced by overexpression of the dominant negative D-PI3-K. These results clearly indicate that PTEN and PI3-K function antagonistically in Drosophila. In this study no PTEN effect on cell migration was observed. Furthermore, signals from the insulin receptor can be antagonized by PTEN function, as in Caenorhabditis elegans and mammals. This argues for a PTEN function as a major conserved negative regulator in the insulin signaling pathway.

Homozygous mutant mice lacking exons 3-5 of the PTEN gene (mPTEN $\left.{ }^{3-5}\right)$ had severely expanded and abnormally patterned cephalic and caudal regions at day 8, 5 of gestation. Embryonic death occurred by day 9, 5 and was associated with defective chorio-allantoic development. Heterozygous mPTEN ${ }^{3-5}$ had an increased incidence of tumours, especially T-cell lymphomas and $\gamma$-irradiation reduced the time lapse of tumour formation. DNA analysis of these tumours revealed the deletion of the mPTEN gene due to loss of heterozygosity $(\mathrm{LOH})$ of the wt allele. Tumours associated with $\mathrm{LOH}$ in mPTEN T-cell lymphomas and teratocarcinomas showed elevated phosphorylation of $\mathrm{PKB} / \mathrm{Akt}$, thus providing a functional connection between mPTEN and protooncogene $\mathrm{PKB} / \mathrm{Akt}$ involved in the development of lymphomas. The PTEN heterozygous $(\mathrm{PTEN}+/-$ ) mice are highly susceptible to the development of various types of tumours. PTEN $+/-$ mice show impaired Fas-mediated apoptosis and may develop lethal autoimmune disorders ${ }^{45}$. It is described PTEN-knockout mouse embryos which display regions of increased proliferation. PTEN-deficient immortalized mouse embryonic fibroblast exhibit decreased sensitivity to cell death, providing in vivo evidence that PTEN negatively regulates cell survival signaling pathways ${ }^{45}$. Surprisingly, as one scales the evolutionary ladder, PTEN plays an increasingly important role. In Caenorhabditis elegans the PTEN homolog is only required for the dauer response to starvation. Analogous to the dauer formation, sporulation in yeast is a developmental state that is activated in response to starvation. The PTEN homolog in yeast plays a role in this process, although it is not essential for the formation of viable spores. The Drosophila PTEN homolog is required for viability and regulation of cell size in eye development. Finally, the inactivation of PTEN in mice results in embryonic lethality and a tendency for the development of tumours in the heterozygous state ${ }^{44}$.

\section{SUMMARY}

Several lines of evidences have confirmed the role of PTEN as a tumour suppressor both in vitro and in vivo.
PTEN participates in the regulation of normal cell growth and survival. Mutation of PTEN directly contributes to cell transformation and neoplastic growth. We have made an effort here to summarise current knowledge of the PTEN molecular structure in relation to its function. PTEN has been demonstrated to be involved in the regulation of a variety of biological phenomena such as cell survival, proliferation or apoptosis. PTEN affects the PKB/Akt pathway by reducing the level of the second messenger Ptd-Ins-3,4,5- $\mathrm{P}_{3}$ cell cycle by upregulation of $\mathrm{p} 27^{\mathrm{Kip} 1}$, cell adhesion as well as migration through regulation of FAK and MAPK activation. Many aspects of the PTEN biology remain unclear and further investigation will be necessary for their elucidation and for practical application of these findings to cancer therapy.

\section{ACKNOWLEDGEMENTS}

We thank L. Popová for help with making of figures. The preparation of this review was supported in part by GACR 204/01/0488, IGA MZ CR NC/6779-3 and MSM 151100001.

\section{REFERENCES}

1. Li J, Yen C, Liaw D, Podsypanina K, Bose S, Wang SI, Puc J, Miliaresis C, Rodgers L, McCombie R, Bigner SH, Giovanella BC, Ittmannn M, Tycko B, Hibshoosh H, Wigler MH, Parsons R. (1997) PTEN, a putative protein tyrosine phosphatase gene mutated in human brain, breast, and prostate cancer. Science 275, 1943-1947.

2. Steck PA, Perhouse MA, Jasser SA, Yung WKA, Lin H, Ligon AZ, Langford LA, Baumgard ML, Haitter T, Davis T, Frye C, Hu R, Swedlund B, Teng DHF, Tavtigian SV. (1997) Identification of a candidate tumour suppressor gene, MMAC1, at chromosome $10 \mathrm{q} 23.3$ that is mutated in multiple advanced cancer. Nat Genet 15, 356-362.

3. Li DM and Sun H. (1997) TEP1, encoded by a candidate tumor suppressor locus, is a novel protein tyrosine phosphatase regulated by transforming growth factor beta. Cancer Res 57, 2124-2129.

4. Huang H, Potter CJ, Tao W, Li DM, Brogiolo W, Hafen E, Sun H, Xu T. (1999) PTEN affects cell size, cell proliferation and apoptosis during Drosophila eye development. Development 126, 5365-5372.

5. Furnari FB, Lin H, Huang HS, Cavenee WK. (1997) Growth suppression of glioma cells by PTEN requires a functional phosphatase catalytic domain. PNAS USA 94, 12479-12484.

6. Mayers MP, Pass I, Batty IH, Kaay JV, Stolarov JP, Hemmings BA, Wigler MH, Downes CP, Tonks NK. (1998) The lipid phosphatase activity of PTEN is critical for its tumor supressor function. PNAS USA 95, 13513-13518.

7. Tamura M, Gu J, Matsumoto K, Aota S, Parsons R, Yamada KM. (1998) Inhibition of cell migration, spreading, and focal adhesions by tumor suppressor PTEN. Science 280, 1614-1617.

8. Besson A, Robbins SM, Yong VW. (1999) PTEN/MMAC1/TEP1 in signal transduction and tumorigenesis, Eur J Biochem 263, $605-611$.

9. Whang YE, Wu X, Suzuki H, Reiter RE, Tran C, Vessella RL, Said JW, Isaacs WB, Sawyers CL. (1998) Inactivation of the tumor suppressor PTEN/MMAC1 in advanced human prostate cancer through loss of expression. PNAS USA 95, 5246-5250.

10. Sano T, Lin H, Chen X, Langford LA, Koul D, Bondy ML, Hess KR, Myers JN, Hong YK, Yung WKA, Steck PA. (1999) Differential expression of MMAC/PTEN in glioblastoma multiforme: relationship to localization and prognosis. Cancer Res 59, 1820-1824. 
11. McMenamin ME, Soung P, Perera S, Kaplan I, Loda M, Seller WR. (1999) Loss of PTEN expression in paraffin-embedded primary prostate cancer correlates with high Gleason score and advanced stage. Cancer Res 59, 4291-4296.

12. Sharrard RM and Maitland N. J. (2000) Alternative splicing of the human PTEN/MMAC1/TEP1 gene. BBA 1494, 282-285.

13. Lee JO, Yang H, Georgescu MM, Cristofano AD, Maehama T, Shi Y, Dixon JE, Pandolfi P, Pavletich NP. (1999) Crystal structure of the PTEN tumor suppressor: implications for its phosphoinositide phosphatase activity and membrane association. Cell 99, 323-234.

14. Barford D, Flint AJ, Tonks NK. (1994) Crystal structure of human protein tyrosine phosphatase 1B. Science 263, 1397-1404.

15. Maehema T and Dixon JE. (1998) The tumor suppressor, PTEN/ MMAC1, dephosphorylates the lipid second messenger, phosphatidylinositol 3, 4, 5-trisphosphate. J Biol Chem 273, 13375-13378.

16. Georgescu MM, Kirsch KK, Akagi T, Shishido T, Hanafusa H. (1999) The tumor suppressor activity of PTEN is regulated by its carboxyl-terminal region. PNAS USA 96, 10182-10187.

17. Leslie NR, Gray A, Pass I, Orchiston EA, Downes CP. (2000) Analysis of the cellular functions of PTEN using catalytic domain and C-terminal mutations: differential effects of C-terminal deletion on signalling pathways. Biochem J 346, 827-833.

18. Wu X, Hepner K, Castelino-Prabhu S, Do D, Kaye MB, Yuan XJ, Wood J, Ross C, Sanwyers CL, Whang YE. (2000) Evidence for regulation of the PTEN tumor suppressor by a membrane-localized multi-PDZ domain containing scaffold protein MAGI-2. PNAS USA 97, 4233-4238.

19. Wu Y, Dowbenko D, Spencer S, Laura R, Lee J, Gu Q, Lasby LA. (2000) Interaction of the tumor suppressor PTEN/MMAC with a PDZ domain of MAGI3, a novel membrane-associated guanylate kinase, J Biol Chem 275, 21477-21485.

20. Adey NB, Huang L, Ormonde PA, Baumgard ML, Pero R, Byreddy DV, Tavtigian SV, Bartel PL. (2000) Threonine phosphorylation of the MMAC1/PTEN PDZ binding domain both inhibits and stimulates PDZ binding. Cancer Res 60, 35-37.

21. Gordon BM, Lu Y, Fang X, Wang H, Eder A, Mai M, Swaby R, Cheng KW, Stokoe D, Siminovitch K, Jaffe R, Gray J. (2001) The role of genetic abnormalities of PTEN and the phosphatidylinositol 3-kinase pathway in breast and ovarian tumorigenesis, prognosis, and therapy. Seminars in Oncology 28, 125-141.

22. Torres J and Pulido R. (2001) The tumor suppressor PTEN is phosphorylated by the protein kinase CK2 at its $\mathrm{C}$ terminus. J Biol Chem 276, 993-998.

23. Stambolic V, Suzuki A, Pompa JL, Brothers GM, Mirtsos C, Sasaki T, Ruland J, Penninger JM, Siderovski DP, Mak TW. (1998) Negative regulation of $\mathrm{PKB} / \mathrm{Akt}$-dependent cell survival by the tumor suppressor PTEN. Cell 5, 29-39.

24. Toker A and Cantley LC. (1997) Signalling through the lipid products of phosphoinositide-3-OH kinase. Nature 387, 673-676.

25. Bellacosa A, Testa JR, Staal SP, Tsichlis PN. (1991) A retroviral oncogene, Akt, encoding a serine-threonine kinase containing an SH2-like region. Science 254, 274-277.

26. Nakatani K, Thompson DA, Barthel A, Sakaue H, Liu W, Weigel RJ, Roth RA. (1999) Up-regulation of Akt3 in estrogen receptordeficient breast cancers and androgen-independent prostate cancer lines. J Biol Chem 274, 21528-21532.

27. Jones PF, Jakubowicz T, Pitosssi FJ, Maurer F, Hemmings BA (1991) Molecular cloning and identification of a serine/threonine protein kinase of the second-messenger subfamily. PNAS USA 88 , 4171-4175.

28. Franke TF, Yang SI, Chan TO, Datta K, Kazlauskas A, Morrison DK, Kaplan DR, Tsichlis PN. (1995) The protein kinase encoded by the Akt proto-oncogene is a target of the PDGF-activated phosphatidylinositol 3-kinase. Cell 81, 727-736.
29. Franke TF, Kaplan DR, Cantley LC. (1997) PI3K: downstream AKTion blocks apoptosis. Cell 88, 435-437.

30. Datta SR, Dudek H, Tao X, Master S, Fu H, Gotoh Y, Greenberg ME. (1997) Akt phosphorylation of BAD couples survival signals to the cell-intrinsic death machinery. Cell 91, 231-241.

31. Cardone MH, Roy N, Stennicke HR, Salvesen GS, Franke TF, Stanbridge E, Frisch S, Reed JC. (1998) Regulation of cell death protease caspase-9 by phosphorylation. Science 282, 1318-1321.

32. Cross DA, Allesi DR, Cohen P, Andjelkovich M, Hemmings BA. (1998) Inhibition of glycogen synthase kinase-3 by insulin mediated by protein kinase B. Nature 378, 785-789.

33. Diehl JA, Cheng M, Roussel MF, Sherr CJ. (1998) Glycogen synthase kinase-3beta regulates cyclin D1 proteolysis and subcellular localization. Genes Dev 12, 3499-3511.

34. Vazquez F, Ramaswamy S, Nakamura N, Sellers R. (2000) Phosphorylation of the PTEN tail regulates protein stability and function, Mol Cell Biol 20, 5010-5018.

35. Ramaswamy S, Nakamura N, Vazquez F, Batt DB, Perera S, Roberts TM, Sellers WR. (1999) Regulation of G1 progression by the PTEN tumor suppressor protein is linked to inhibition of the phosphatidylinositol 3-kinase/Akt pathway. PNAS USA 96, 2110-2115.

36. Chung JK, Grammer TC, Lemon KP, Kazlauskas K, Blenis J. (1994) PDGF- and insulin-dependent pp70S6k activation mediated by phosphatidylinositol-3-OH kinase. Nature 370, 71-75.

37. Hlobilkova A, Guldberg P, Thullberg M, Zeuten J, Lukas J, Bartek J. (2000) Cell cycle arrest by the PTEN tumour suppressor is target cell specific and may require protein phosphatase activity. Exp Cell Res 256, 571-577.

38. Weng LP, Brown JL, Eng C. (2001) PTEN coordinates G(1) arrest by down-regulating cyclin D1 via its protein phosphatase activity and up-regulating p27 via its lipid phosphatase activity in a breast cancer model. Hum Mol Genet 10, 599-604.

39. Cheney IW, Neuteboom STC, Vaillancourt MT, Ramachandra M, Bookstein R. (1999) Adenovirus-mediated gene transfer of MMAC1/PTEN to glioblastoma cells inhibits $\mathrm{S}$ phase entry by the recruitment of p27Kip1 into cyclin $\mathrm{E} / \mathrm{CDK} 2$ complexes. Cancer Res 59, 2318-2323.

40. Weng LP, Brown JL, Baker KM, Ostrowski MC, Eng C. (2002) PTEN blocks insulin-mediated ETS-2 phosphorylation through MAP kinase, independently of the phosphoinositide 3-kinase pathway. Hum Mol Genet 11, 1687-1696.

41. Gu J, Tamura M, Yamada KM. (1998) Tumor suppressor PTEN inhibits integrin-and growth factor-mediated mitogen-activated protein (MAP) kinase signaling pathways. J Cell Biol. 143, 1375-1383.

42. Gu J, Tamura M, Pankov R, Danen EHJ, Takina T, Matsumoto K, Yamada KM. (1999) Shc and FAK differentially regulate cell motility and directionality modulated by PTEN. J Cell Biol 146, 389-403.

43. Mihaylova VT, Borland C, Manjarrez L, Stern MJ, Sun H. (1999) The PTEN tumor suppressor homolog in Caenorhabditis elegans regulates longevity and dauer formation in an insulin receptorlike signaling pathway. PNAS USA 96, 7427-7432.

44. Heymont J, Berenfeld L, Collins J, Kaganovich A, Maynes B, Moulin A, Ratskovskaya I, Poon PP, Johnson GC, Kamenetsky M, DeSilva J, Sun H, Petsko GA, Engebrecht J. (2000) TEP1, the yeast homolog of the human tumor suppressor gene PTEN/ MMAC1/TEP1, is linked to the phosphatidylinositol pathway and plays a role in the developmental process of sporulation. PNAS USA 97, 12672-12677.

45. Suzuki A, Pompa JL, Stambolic V, Elia AJ, Sasaki T, Barrantes IB, Ho A, Wakeham A, Itie T, Khoo W, Fukumoto M, Mak TW. (1998) High cancer susceptibility and embryonic lethality associated with mutation of the PTEN tumor suppressor gene in mice, Curr Biol 8, 1169-1178. 